\title{
A Importância do Fator Motivacional no Processo Ensino- Aprendizagem de Algoritmos e Lógica de Programação para Alunos Repetentes
}

\author{
Antunes Santos, Arthur Gorgônio, Amarildo Lucena, Flavius Gorgônio \\ Laboratório de Inteligência Computacional Aplicada a Negócios (LABICAN) \\ Universidade Federal do Rio Grande do Norte (UFRN) \\ Rua Joaquim Gregório, S/N - Penedo - 59.300-000 - Caicó - RN \\ antunesbsi@gmail.com, arthur@gorgonio.com.br, \{infoslave,flgorgonio\}@gmail.com
}

\begin{abstract}
A substantial proportion of freshmen in Information Technology graduation courses face difficulties in classes during the two initial semesters, which causes some of these students to end up failing. If these classes do not use alternative teaching methodologies that encourage and contribute to the student's recovery of credits in the course, the discouragement may cause new failures in the following semesters and contribute to increased dropout rate. This paper presents promising results of a practical experience using Arduino platform and visual programming, with a group of repeating students of the Algorithms and Programming Logic class of the first semester in the Information Systems course at the Federal University of Rio Grande do Norte.
\end{abstract}

Resumo. Uma parte considerável dos ingressantes em cursos superiores na área de Tecnologia da Informação enfrenta dificuldades nas disciplinas de programação dos primeiros semestres, sendo que alguns desses alunos acabam sendo reprovados. Se tais disciplinas não utilizam metodologias de ensino alternativas que estimulem e contribuam para a recuperação do aluno, a desmotivação pode ocasionar novas reprovações nos semestres seguintes $e$, por conseguinte, contribuir para o aumento da evasão. $O$ presente trabalho apresenta resultados promissores de uma experiência prática de uso da plataforma Arduino e da programação visual em uma turma de alunos repetentes na disciplina de algoritmos e lógica de programação do primeiro semestre do curso de Sistemas de Informação da UFRN.

\section{Introdução}

Várias disciplinas presentes nos cursos da área de TI - Tecnologia da Informação (Computação, Informática e áreas afins) apresentam a programação de computadores como foco central, assunto que permanece, ao longo do curso, de forma recorrente. $\mathrm{O}$ aprendizado desses conceitos já nas disciplinas do primeiro semestre visa tornar o indivíduo apto a utilizar a lógica de programação como ferramenta para a resolução de diversos problemas computacionais, fator importante e necessário para disciplinas mais avançadas. Porém, diversas pesquisas apontam as dificuldades encontradas nesse processo de aprendizagem, sendo comum a existência de enormes taxas de insucesso em disciplinas iniciais de programação, onde os aspectos básicos de algoritmos e lógica de programação são ensinados [Gomes, Henriques e Mendes 2008] [Campos 2010] [Peixoto et al. 2013]. 
O aprendizado desses conceitos exige do aluno o domínio de habilidades específicas relacionadas à abstração e ao raciocínio lógico, aptidões que são pouco exploradas no ensino médio. Pereira Júnior e Rapkiewicz (2004) sustentam que as disciplinas de algoritmos e lógica de programação são as que apresentam maior número de reprovados dentre as ciências exatas, a despeito do fato de que são as mais importantes na formação do aluno que ingressa em um curso superior nesta área. Ademais, o desconhecimento dos objetivos e do perfil profissional exigidos pelo curso têm gerado desmotivação em uma parcela significativa dos alunos ingressantes e, via de regra, também contribuem significativamente para o aumento dos altos índices de reprovação e da taxa de evasão.

Esse fato, aliado a uma diminuição na procura por cursos na área de TI ocorrida nos últimos anos já preocupa os especialistas, haja visto que esta tem sido um realidade comum em grande parte das instituições de ensino, tanto no Brasil, quanto em outras partes do mundo [Caitlin e Randy 2007]. Face a essas dificuldades, pesquisadores da área buscam identificar limitações e propor melhorias no processo ensino-aprendizagem de algoritmos e lógica de programação através do uso de atividades práticas, elemento necessário para sustentar o entusiasmo e a disposição no aprendizado de programação de computadores.

Dessa forma, ao mesmo tempo em que é necessário realizar estudos que contribuam para o levantamento de informações sobre as causas que levam ao fracasso e à desmotivação do aluno pelo curso, é essencial a realização de estudos experimentais que permitam validar novas estratégias de ensino, oferecendo uma oportunidade de utilizar a tecnologia para explorar a criatividade dos alunos nas diferentes possibilidades de construir sistemas, seja através de técnicas lúdicas ou conectando dispositivos simples diretamente a componentes de hardware e software.

Analisando essas questões sob uma visão pedagógica, que identifica a aprendizagem como um processo de construção de conhecimento, surge a seguinte questão: "seria possível manter o interesse do aluno nos conteúdos apresentados por meio de estratégias práticas adequadas que o motivassem e contribuíssem para a melhoria do ensino de conceitos de algoritmos e lógica de programação?". Nessa linha de pesquisa, este trabalho avalia a utilização de uma estratégia motivacional junto a uma turma de alunos repetentes do curso de Sistemas de Informação da UFRN, com a finalidade de manter o interesse no aprendizado durante a disciplina BSI1101 Algoritmos e Lógica de Programação (ALP). Essa estratégia realizou intervenções metodológicas através da inclusão de atividades práticas complementares, inseridas ao final de algumas aulas, utilizando recursos de programação visual e da plataforma Arduino como ferramentas auxiliares ao processo ensino-aprendizagem.

\section{Fundamentação Teórica}

\subsection{Dificuldades no ensino de programação}

Nas pesquisas realizadas sobre o ensino da programação, observa-se uma preocupação crescente sobre as dificuldades com o processo de ensino-aprendizagem de algoritmos e lógica de programação [Pereira Júnior e Rapkiewicz 2004]. Os estudos são motivados, sobretudo, pela importância dos conceitos de programação na vida acadêmica dos cursos de computação. A partir da preocupação com o processo de adequação dos 
alunos ao raciocínio lógico exigido pela lógica de programação, outros pesquisadores passaram a perceber a necessidade de conceitos e técnicas utilizadas na concepção do raciocínio ainda no nível médio [Santos e Costa 2006].

A resistência perante a condução do aprendizado lógico se forma antes do começo das atividades, fato que pode ocorrer por sua característica lógico-matemática, dado o enraizado estigma relacionado aos conteúdos não abordados no nível médio. Dessa forma, o professor passa a necessitar de uma estratégia pedagógica adequada que contribua para o aprendizado, o que acaba deixando claro que a absorção do conteúdo passado em sala de aula não depende apenas do nível de abstração do aluno.

O aluno se torna mais interessado em um ambiente descontraído e colaborativo do que em um ambiente instrucionista, no qual o professor preocupa-se em mostrar conhecimento [Pereira Júnior e Rapkiewicz 2004]. Por outro lado, o desinteresse do aluno pelo conteúdo apresentado pode ser ainda maior em situações onde o aluno é repetente e está revendo conceitos que já foram abordados em semestres anteriores. Assim, é esperado que a metodologia utilizada em disciplinas que contenham um grande número de repetentes seja um fator importante para a motivação. Dessa forma, a utilização de uma metodologia diferenciada e pedagogicamente adequada para turmas de repetentes mantém o aluno motivado no aprendizado do conteúdo.

\subsection{Recursos lúdicos no aprendizado da programação de computadores}

Almeida (1998) considera a educação lúdica como responsável pela ação de buscar e de apropriar-se dos conhecimentos dos alunos exigindo esforço, participação, indagação, criação, reflexão e socialização com prazer. Outro aspecto relevante sobre a educação lúdica, é que esta integra a teoria com a prática atuante, com o principal objetivo de fazer do ato de educar, um compromisso conscientemente intencional e de esforço, sem perder o caráter de prazer e satisfação. Em direção semelhante, a proposta defendida por este trabalho tem como apoio à ludicidade para minimizar as dificuldades dos alunos em ALP por meio de ações concretas, experimentações e reflexões aplicadas ao Arduino e a programação visual, tendo como base metodológica os princípios da Aprendizagem Baseada em Projetos (PBL).

O Arduino é uma plataforma computacional de código aberto que tem como principal característica a simplicidade de uso. Composto por uma placa física, composta por um microcontrolador e várias portas de entrada/saída, a plataforma possui uma interface serial ou USB para ser interligada ao computador, o que permite ao usuário programá-la e interagir em tempo real com a execução do programa [Banzi 2011]. A plataforma Arduino suporta várias linguagens de programação, entre elas a linguagem Processing, desenvolvida especialmente para produção de artes eletrônicas e para o desenvolvimento de projetos visuais, ensinando noções básicas de programação a um público pouco acostumado com as linguagens tradicionais.

O Scratch ${ }^{1}$ é um ambiente de ensino de programação desenvolvido pelo MIT Massachusetts Institute of Technology e especialmente destinado ao ensino de programação para crianças. $\mathrm{O}{\mathrm{S} 4 \mathrm{~A}^{2}}^{2}$ (Scratch for Arduino) é uma modificação do

\footnotetext{
${ }^{1}$ http://www.scratch.mit.edu/

${ }^{2}$ http://www.s4a.cat/
} 
Scratch que permite a programação da plataforma Arduino de forma simples, tornando possível gerenciar, de forma simples, sensores e atuadores conectados ao Arduino através de uma linguagem de programação baseada em blocos. O projeto foi desenvolvido em 2010 pela Equipe de Smalltalk do Citilab para incentivar um maior número de pessoas a aprender a programar através de uma interface de alto nível para programadores que utilizam a plataforma Arduino.

O desenvolvimento da PBL teve suas origens por volta de 1900, quando o filósofo americano John Dewey comprovou o "aprender mediante o fazer", valorizando a aprendizagem experiencial, prática e dirigida pelo aluno em uma forma gradativa de aquisição de conhecimento relativo para resolver situações reais em projetos referentes aos conteúdos na área de estudos. Esta ideia reflete no processo de aprendizagem estruturado em atividades iniciadas pela apresentação do problema aos estudantes sem que eles tenham tido, anteriormente, acesso às informações necessárias para abordá-lo. Ou seja, esta metodologia transfere a responsabilidade da aprendizagem do professor para o estudante, onde o estudante passa a ser o principal gerador de conhecimento ao procurar ativamente a informação que necessita para resolver um determinado problema [Markham, Larmer e Ravitz 2008].

\section{Ambiente de Estudo e Metodológica Utilizada}

\subsection{Cenário atual do curso de Sistemas de Informação da UFRN}

O curso de Bacharelado em Sistemas de Informação (BSI) da Universidade Federal do Rio Grande do Norte (UFRN) teve inicio no ano de 2009, sendo oferecido pelo Centro de Ensino Superior do Seridó (CERES), no Campus de Caicó, interior do Rio Grande do Norte. O curso tem duração mínima de quatro anos e as aulas ocorrem nos turnos matutino e vespertino. O curso visa a formação de profissionais aptos a compreender as necessidades sistêmicas das organizações e transformar essas necessidades em soluções de tecnologia da informação. A cada ano o curso recebe 50 novos alunos, sempre no primeiro semestre letivo, sendo o ENEM/SISU a principal forma de ingresso.

Apesar de ter apenas uma entrada anual, a disciplina ALP tem sido oferecida todos os semestres desde a criação do curso em 2009. Mesmo sendo um componente curricular vinculado ao primeiro período, a necessidade de oferta dessa disciplina em todos os períodos se dá pelo constante volume de alunos repetentes, alguns deles, por várias vezes consecutivas. Desde 2010.2, o curso adota Python como linguagem de programação inicial, em função da sua sintaxe enxuta e facilidade de aprendizado.

A Figura 1 apresenta um levantamento realizado sobre a situação dos alunos nos últimos cinco anos e meio do curso de BSI do CERES/UFRN em relação à disciplina ALP. As barras verticais no gráfico correspondem aos períodos de ingressantes no curso e as barras horizontais correspondem às turmas apenas com repetentes, assim é possível observar uma crescente taxa de insucesso desde o período de 2009.1 até 2013.1. É importante ressaltar que os trancamentos e evasões ocorridas na disciplina também contam como taxas de insucessos apresentadas na Figura 1. Conforme informações obtidas a partir do Sistema de Controle Acadêmico da UFRN e fornecidas pelos próprios alunos durante o processo de trancamento, a maioria dos trancamentos realizados no período teve como principal motivo a dificuldade no acompanhamento da disciplina e a falta de identificação com a metodologia adotada nas aulas. 


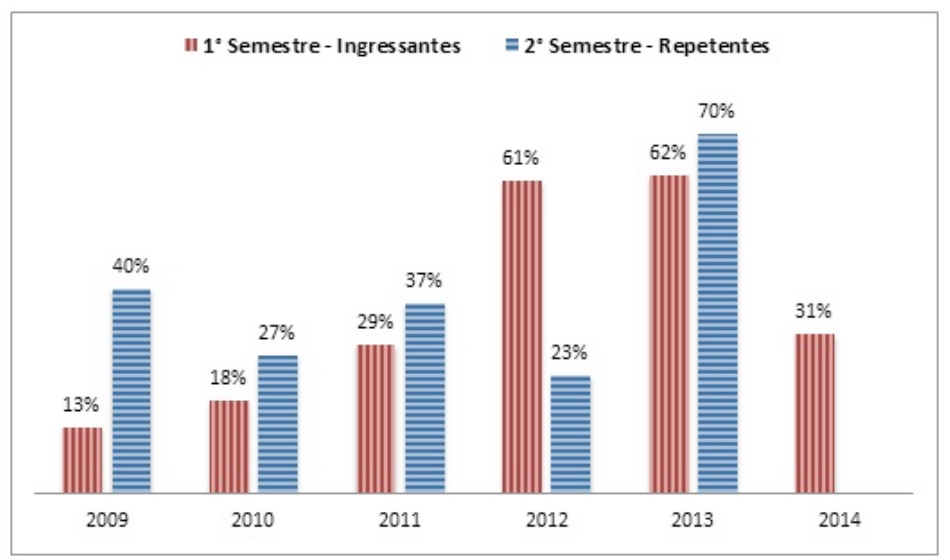

Figura 1. Percentuais de insucesso, por período, nas turmas da disciplina ALP

\subsection{Experiência prática}

A metodologia aplicada neste estudo apresentou uma abordagem alternativa aos métodos tradicionais de ensino, com finalidade motivacional, que combinou recursos de programação visual e a utilização de uma plataforma de hardware Arduino, além de sensores de fácil aplicação, para atuarem como facilitadores no processo de ensinoaprendizagem, visando manter o interesse e o nível de satisfação de cada aluno no desenvolvimento de algoritmos através do uso dessas ferramentas. Além da linguagem Python, já utilizada nas aulas convencionais, foram apresentados aos alunos exemplos de códigos em linguagem $C$, em pseudocódigo e na linguagem visual S4A.

Diante da proposta da metodologia de utilizar recursos motivacionais, o ambiente de programação foi uma das principais mudanças exigidas na forma de utilização dos conceitos de programação. Em primeiro lugar, pela necessidade de um ambiente visual que oferecesse interface de fácil utilização, possibilitando ao aluno com dificuldades (por serem repetentes) uma forma mais intuitiva de desenvolvimento de programas. Em segundo lugar, pela falta de uma ferramenta que estivesse à disposição do professor por meio da qual fosse possível demonstrar na prática muitos dos conceitos teóricos, permitindo ao aluno nesse processo de construção do conhecimento, a oportunidade de participar de uma aprendizagem mais efetiva. Além disso, a metodologia utilizando programação visual e a plataforma Arduino foi baseada na proposta da PBL, uma vez que esta metodologia de ensino se baseia no aprendizado autônomo do aluno, utilizando múltiplos meios e formas de absorver o conhecimento.

Através da metodologia proposta, foi possível avaliar experimentalmente a utilização da programação visual e do Arduino como ferramentas para o incentivo à programação de computadores. A condução para apresentação da definição e do uso dessas ferramentas em pequenos intervalos no final das aulas de ALP foi essencial para a posterior avaliação experimental à qual a metodologia proposta foi submetida. Após o conteúdo visto durante a semana, os alunos eram apresentados a diferentes experiências de utilização do Arduino para as mais diversas formas de utilização da ferramenta no mundo real, tornando o conteúdo prático e agradável.

As primeiras intervenções metodológicas tiveram como objetivo apresentar a programação visual e a plataforma Arduino. Assim, o protótipo de um veículo autônomo foi mostrado aos alunos (Figura 2), juntamente com a apresentação de alguns 
dos recursos disponíveis para controle do veículo. Este protótipo foi construído a partir de uma placa Arduino montada em um chassi com rodas motorizadas e um sensor de distância. A apresentação de conceitos básicos sobre a montagem de circuitos e dos módulos que compõem o veículo foi fundamental para proporcionar um primeiro contato dos estudantes com as ferramentas que seriam utilizadas nas aulas seguintes.

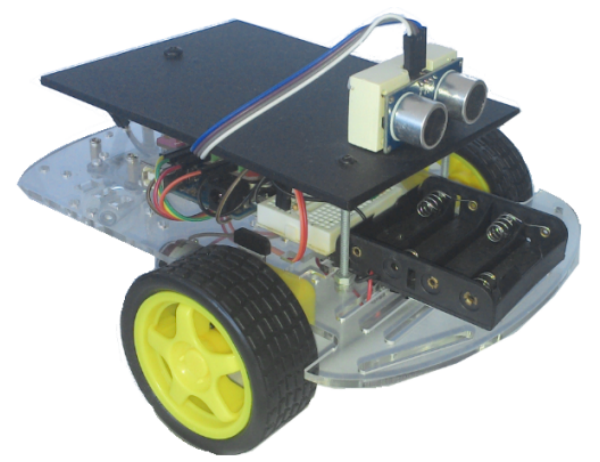

Figura 2. Protótipo do veículo autônomo utilizado durante as aulas

Nas intervenções seguintes, foi incentivado o uso de estruturas de decisão para o desenvolvimento de uma solução para movimentar o veículo através de um conjunto de obstáculos. A partir dos conceitos apresentados na aula teórica e do entendimento básico sobre o funcionamento das instruções condicionais de decisão, a metodologia proposta buscou trabalhar o raciocínio lógico dos alunos na busca por uma solução algorítmica que utilizasse os dados coletados a partir dos sensores interligados ao protótipo para movimentar o veículo apresentado na Figura 2.

Assim, em uma das aulas foi solicitado aos alunos um algoritmo que ajudasse o veículo a se movimentar, de forma autônoma, em um ambiente com obstáculos. Para essa atividade, considerou-se uma simulação de uma situação real, onde o veículo estaria em um terreno plano, cercado por três paredes e com apenas uma saída. Uma ilustração para o problema e uma proposta de algoritmo estão apresentados na Figura 3. $\mathrm{O}$ algoritmo final envolveu uma série de condições de decisão para verificar a distância do veículo à medida que ele se movimentava para frente. Dependendo da existência de algum obstáculo, o veículo deveria fazer uma nova leitura de possíveis caminhos livres ao seu redor utilizando os sensores de distância acoplados.
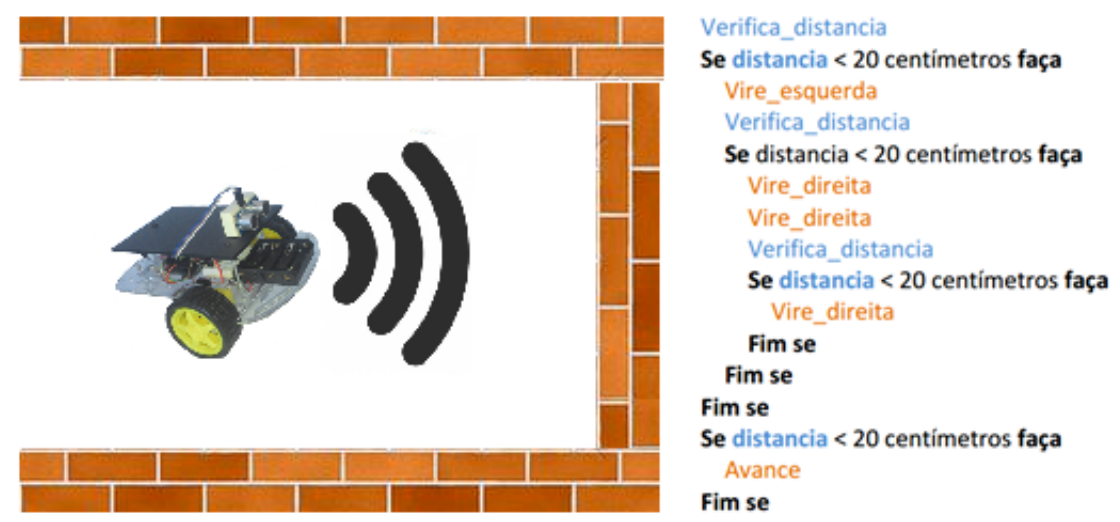

Figura 3. Exemplo de problema para ensino de estruturas de decisão 
Acompanhando a evolução gradual do conteúdo que era repassado aos alunos pelo professor da disciplina, nas intervenções metodológicas seguintes foram elaborados exemplos para que os estudantes pudessem exercitar o raciocínio lógico através do uso de estruturas de repetição. Em uma das aulas, a proposta foi contribuir para que o aluno pudesse compreender a execução da repetição de um bloco de instruções enquanto uma condição fosse satisfeita. Para isso, foi utilizado o mesmo veículo autônomo dos exemplos anteriores, porém com a utilização de uma conexão bluetooth para torná-lo controlável a partir do software BT Joystick que era executado a partir de um smartphone.

A Figura 4 mostra a tela principal do BT Joystick, aplicativo que simula um joystick e que pode ser conectado ao veículo através de um módulo bluetooth ligado ao Arduino. Assim, é possível enviar comandos para controlar a direção ao veículo através do envio de caracteres de texto via bluetooth. O exemplo incluiu, além das estruturas de repetição para leitura sucessiva dos comandos recebidos, a utilização de instruções de decisão para determinar qual a direção a ser seguida pelo veículo. Nesse exemplo, foram utilizadas ainda, estruturas de decisão aninhadas, uma das principais dificuldades dos alunos segundo o professor da disciplina.

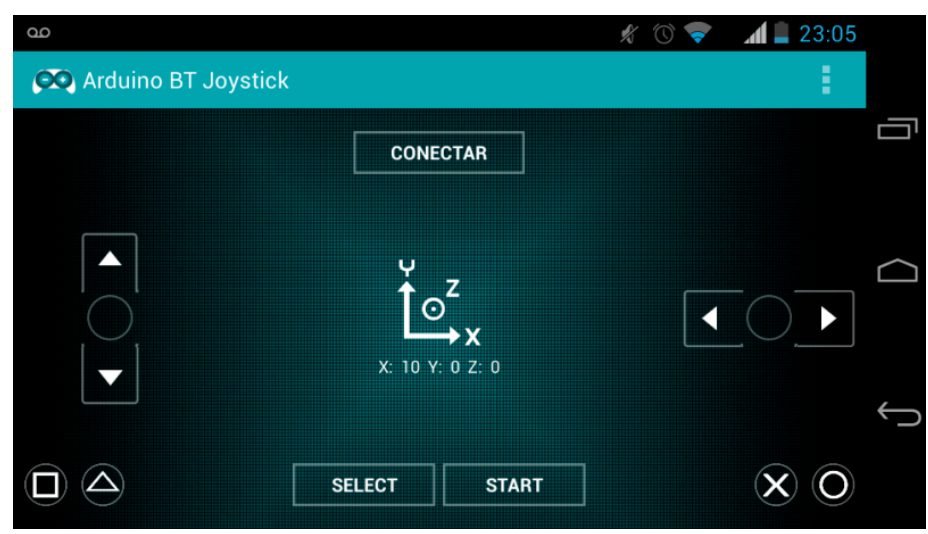

Figura 4. Tela principal do aplicativo BT Joystick

Visando mudar o cenário de aprendizado, até então baseados apenas no uso do veículo já montado, e buscando incentivar os alunos participantes da proposta metodológica a despertarem novas ideias e formas de abstração, outros elementos foram apresentados nas intervenções metodológicas seguintes. Durante a abordagem prática, foram apresentados novos componentes eletrônicos, dentre eles, um relê, um sensor de presença e uma lâmpada comum. Com esses novos elementos eletrônicos foi elaborado um sistema para controle de acendimento de uma lâmpada apenas quando movimentos fossem detectados em um espaço físico delimitado. O sistema foi implementado e explicado aos estudantes usando o código fonte do próprio Arduino e a montagem de blocos de códigos no S4A.

Ainda durante essa etapa, foram discutidas outras possíveis aplicações para utilização de sensores de presença e de relês, tais como sistemas de alarmes e sistemas para ligação e desligamento automático de eletrodomésticos. Na Figura 5, é apresentado o código fonte de um dos experimento, tanto na linguagem do Arduino quanto utilizando a linguagem de blocos do S4A. Ambos os códigos apenas verificam o estado do sensor de presença e liberam o acendimento da lâmpada caso seja detectado algum movimento no ambiente. 


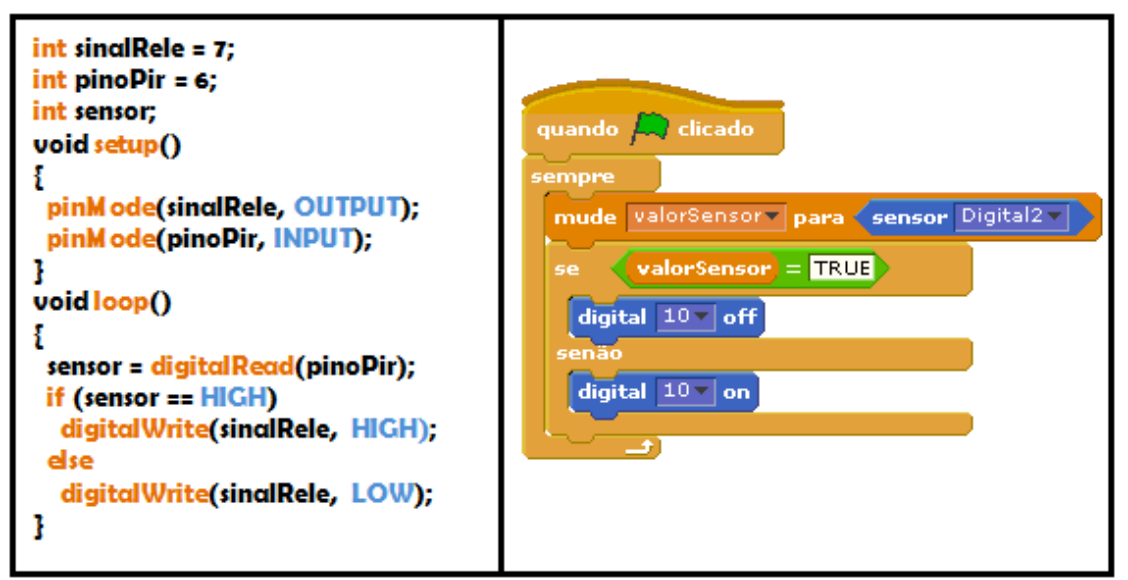

Figura 5. Comparação entre a linguagem do Arduino e do ambiente S4A

É importante deixar claro que o uso da robótica nas intervenções não teve como objetivo direcionar os alunos para a montagem de robôs e de outros sistemas autônomos. Ao invés disso, o objetivo principal dessas intervenções foi servir como ferramenta para auxiliar no aprendizado de lógica de programação, facilitando a assimilação dos conceitos teóricos vistos em sala de aula. Durante as intervenções, foi possível mostrar o potencial que os estudantes possuem para conduzir seu próprio aprendizado. Além disso, foi possível verificar ainda que os alunos se adaptaram bem às diferentes linguagens utilizadas e que a compreensão dos conceitos fundamentais relacionados ao processo de construção de algoritmos independe da linguagem adotada.

\section{Resultados Obtidos}

Face às inúmeras variações nas formas de avaliação, diferentes professores e diversidade de metodologias de ensino adotadas entre os diferentes semestres, considerou-se que uma avaliação quantitativa não seria um critério suficientemente justo para comparar o desempenho da metodologia proposta frente às demais abordagens adotadas anteriormente. Por isso, a avaliação da metodologia se deu de forma qualitativa, a partir da aplicação de questionários junto aos alunos do semestre 2014.2, todos repetentes, que foram submetidos às intervenções metodológicas. Mesmo diante da pouca quantidade de alunos, uma vez que apenas 16 alunos matricularam-se na disciplina nesse período, os resultados foram bastante significativos no que se diz respeito à aceitação da metodologia.

Aproveitando-se o primeiro encontro com a turma, foi aplicado um questionário com o objetivo de avaliar a metodologia e o professor do semestre anterior (em 2014.1), a fim de identificar as principais dificuldades com a disciplina de ALP. Os resultados obtidos no questionário I apresentaram uma boa avaliação para a metodologia adotada, professor e acompanhamento dos monitores durante a oferta anterior da disciplina. Importante frisar que, diante da pesquisa, os alunos consideraram o conteúdo abordado na disciplina como sendo "razoavelmente difícil", o que demonstra a dificuldade que os alunos enfrentam no aprendizado deste conteúdo. Por outro lado, verificou-se uma aceitação positiva à utilização de novas estratégias, conforme todos pontuaram, além de um grande interesse em participar do experimento com o uso da nova metodologia.

Após as quatro etapas de intervenções realizadas com os alunos, um novo questionário foi aplicado para avaliar os pontos positivos e negativos da proposta 
metodológica. Nesse questionário, os alunos fizeram uma boa consideração ao uso da metodologia e da utilização de exercícios práticos após a exposição de conceitos teóricos em aula. O caráter motivacional das aulas práticas atuou de forma significativa para aumentar o interesse dos alunos pelo conteúdo, vários alunos afirmaram ter despertado o interesse pela plataforma Arduino e programação visual, o que os levou a realizar pesquisas avançadas sobre o assunto, além de contribuir para aumentar sua curiosidade em projetar seus próprios veículos robôs utilizando o Arduino e sensores.

Embora não haja evidências imediatas que vinculem a melhoria do rendimento escolar no semestre à utilização em sala de aula da abordagem proposta, o rendimento individual (medido a partir de suas notas) de $62 \%$ dos alunos repetentes aumentou em relação ao semestre anterior e o índice de aprovação atingiu 50\%. Por esta razão, visando avaliar melhor o nível de interesse e motivação dos alunos durante as intervenções metodológicas, foram realizadas algumas entrevistas adicionais baseadas em um roteiro de perguntas abertas. Todos os entrevistados afirmaram ter adquirido mais interesse e motivação quando os exemplos eram apresentados na plataforma Arduino. Um dos alunos ressaltou a importância de ver o algoritmo sendo executado e transformado em uma ação física, tornando a atividade mais interessante.

Durante o desenvolvimento da entrevista, buscou-se ainda mensurar a influência da linguagem de programação utilizada no desenvolvimento das atividades. Apesar da maioria dos estudantes terem respondido no questionário que preferiam a linguagem Python, por esta ser mais fácil de usar do que a linguagem $\mathrm{C}$, durante a entrevista os alunos citaram que isso é devido a familiaridade já adquirida com a linguagem Python no semestre anterior. Apesar disso, os alunos tem consciência que o domínio da linguagem $\mathrm{C}$ é essencial para a área, uma vez que é utilizada em outras disciplinas ao longo do curso. Além disso, a linguagem visual do ambiente S4A também teve boa aceitação, embora haja a percepção de que o objetivo principal da disciplina não é focar em uma ou outra linguagem, mas nos conceitos elementares da lógica de programação.

Dentre as dificuldades identificadas na metodologia, alguns alunos afirmaram não terem conseguido assimilar todos os conteúdos repassados durante as aulas teóricas para ser utilizado posteriormente nos experimentos, sugerindo a necessidade de interação simultânea entre teoria e prática. A maioria destacou ainda que a linguagem $\mathrm{C}$ não era tão intuitiva quanto a linguagem Python, o que leva a crer que a utilização de mais de uma linguagem de programação nos experimentos influenciou na assimilação dos conteúdos. Conforme esperado, a não disponibilidade de recursos para que todos pudessem criar seus próprios projetos práticos foi um dos pontos mais comentados na entrevista. Todos os alunos questionaram a possibilidade de terem disponíveis kits Arduino e outros componentes eletrônicos para serem trabalhados individualmente. Alguns justificaram ainda que, se houvessem mais aulas práticas durante a disciplina, poderiam compreender mais facilmente os conteúdos apresentados.

\section{Considerações Finais e Conclusão}

Tendo em vista os resultados obtidos com o estudo realizado, considera-se que os objetivos inicialmente definidos foram alcançados. Com o uso do Arduino e do S4A foi possível explorar os conceitos computacionais de raciocínio lógico na disciplina de ALP. Apesar de não ter sido possível avaliar os conteúdos continuamente, esta metodologia de ensino poderá ser adaptada a outros assuntos vistos ao longo da 
disciplina ALP, tendo em vista a variedade de experimentos possíveis com o S4A e com a possibilidade de trabalhar todos os assuntos da linguagem $C$ na plataforma Arduino.

Embora satisfatórios e motivadores, os resultados deste trabalho não são estatisticamente significativos a ponto de serem generalizados para assegurar o sucesso da abordagem utilizada. Tal processo exigiria um acompanhamento mais criterioso, preferencialmente de várias turmas, juntamente com um planejamento mais cauteloso dos experimentos. Este trabalho sugere apenas que a utilização da abordagem proposta junto a alunos repetentes influencia de forma significativa para que os alunos tenham um desempenho mais satisfatório do ponto de vista motivacional do que utilizando uma abordagem tradicional.

Sendo assim, apesar das dificuldades apresentadas neste trabalho, as vantagens superaram os desafios no sentido que os alunos não apenas aprenderam princípios básicos de programação, mas desenvolveram novas habilidades de assimilação de conteúdo, exercitaram o desenvolvimento de atividades em grupo e foram incentivados a desenvolverem sua criatividade para resolver problemas. Em trabalhos futuros, pretende-se validar a metodologia usando uma amostra maior de alunos, além de experimentar a aplicação da metodologia em outras disciplinas do curso. Também está prevista a aquisição de kits Arduino para a experimentação prática em uma quantidade maior de aulas e com a participação mais efetiva dos alunos na montagem dos circuitos.

\section{Referências}

Almeida, P. N. (1998) Educação lúdica: Técnicas e jogos pedagógicos, Edições Loyola.

Banzi, M. (2011) Primeiros Passos com o Arduino, Novatec.

Caitlin K., Randy P. (2007) Using storytelling to motivate programming. Communications of the ACM. v. 50, n. 7, p. 58-64.

Campos, R. (2010) Metodologia ERM2C: Para melhoria do processo de ensinoaprendizagem de lógica de programação. In Anais do XVIII WEI. SBC.

Gomes, A., Henriques, J., Mendes, A. (2008) Uma proposta para ajudar alunos com dificuldades na aprendizagem inicial de programação de computadores. In Educação, Formação \& Tecnologias, v.1, n.1, p. 93-103. EDUCOM - APTE.

Markham, T., Larmer, J. e Ravitz, J. (2008) Aprendizagem baseada em projetos: Um guia para professores de ensino fundamental e médio, Artmed, $2^{a}$ edição.

Peixoto, M., Scaico, P., Souza, F., Peixoto, H. (2013) Uso de Estratégias de Aprendizagem e Motivacionais pelos Alunos em Disciplinas de Programação: Um Estudo de Caso na Licenciatura em Computação. In: Anais do XXI WEI, p. 460-465. SBC.

Pereira Júnior, J., Rapkiewicz, C. (2004) O Processo de Ensino-Aprendizagem de Fundamentos de Programação: Uma visão crítica da pesquisa no Brasil. In Anais do I WEI. Sociedade Brasileira de Computação.

Santos, R., Costa, H. (2006) Análise de Metodologias e Ambientes de Ensino para Algoritmos, Estruturas de Dados e Programação aos Iniciantes em Computação e Informática. INFOCOMP Journal of Computer Science. v. 5, p. 41-50. 